\title{
Optimal Torque Control for an Electric-Drive Vehicle with In- Wheel Motors: Implementation and Experiments
}

\author{
Abtin Athari \\ Univ. of Waterloo \\ Saber Fallah \\ Univ. of Surrey \\ Bin Li and Amir Khajepour \\ Univ. of Waterloo \\ Shih-Ken Chen and Baktiar Litkouhi \\ General Motors Company
}

\begin{abstract}
This paper presents the implementation of an off-line optimized torque vectoring controller on an electric-drive vehicle with four in-wheel motors for driver assistance and handling performance enhancement. The controller takes vehicle longitudinal, lateral, and yaw acceleration signals as feedback using the concept of state-derivative feedback control. The objective of the controller is to optimally control the vehicle motion according to the driver commands. Reference signals are first calculated using a driver command interpreter to accurately interpret what the driver intends for the vehicle motion. The controller then adjusts the braking/throttle outputs based on discrepancy between the vehicle response and the interpreter command. A test vehicle equipped with four in-wheel electric motors, vehicle sensors, communication buses, and DSPACE rapid prototyping hardware is instrumented and the control performance is verified through vehicle handling tests under different driving conditions.
\end{abstract}

CITATION: Athari, A., Fallah, S., Li, B., Khajepour, A. et al., "Optimal Torque Control for an Electric-Drive Vehicle with In-Wheel Motors: Implementation and Experiments," SAE Int. J. Commer. Veh. 6(1):2013, doi:10.4271/2013-01-0674.

\section{INTRODUCTION}

Increasing environmental and safety concerns, along with rising gasoline prices, are driving a worldwide shift in the automotive industry towards the development of green intelligent transportation systems. Today, electric vehicles (EVs) are seen as the leading contender to displace existing fleets of gasoline-powered cars. EVs allow for the possibility of zero greenhouse-gas and air-pollutant emissions as well as very little noise pollution. Another important advantage of electric vehicles over the conventional internal combustion engines (ICEs) is the very high energy efficiency and relatively low cost of the electric motor. The main concern currently being faced is the low energy and power densities of batteries compared to the liquid fuels.

EVs use electric motors to propel the car and use batteries to store electricity. Four in-wheel motors, two independent motors for the front and rear axles, and a single motor running all four wheels are possible motor configurations for shaping the propel system. The rapid dynamics of the motors provide the opportunity to accurately control the wheel speeds, thereby achieving better handling performance. In addition, this property allows stability and safety controllers such as active cruise control, collision avoidance, and emergency brake assist to apply their commands much faster, resulting in better incident prevention. Motors in EVs also help in stabilizing vehicle motion by generating counterdirectional torques between the left and right wheels using control strategies such active software differentials, active brake bias, and brake steer. While applying these controllers on ICEVs requires sophisticated hardware components that add complexity to the vehicle structure, the application of such controllers in EVs can be carried out using only software modification. 
Substantial research has been carried out on the development of electric vehicles and related control strategies during the last two decades. For example, Ref. [1] provides an overview of electric-vehicle technology while Refs. [2] and [3] report on the application of different stability controllers in electric vehicles. Moreover, a combination of direct yaw-moment and active front steering controllers for electric vehicles with four in-wheel motors is proposed as a stability control system in Ref [4]. A torque-control distribution for an electric vehicle is presented in Ref [ $[5]$ that takes advantage of front-wheel drive, rear-wheel drive, and all-wheel drive configurations in vehicles. The applications of electric braking control systems and traction control systems in electric vehicles are presented in Refs. [6] and [7], respectively, while Ref. [] presents a nonlinear optimal theory used for controlling the speed of light-weighted allelectric vehicles. Refs. [9] to [10] discuss the effectiveness of their proposed direct yaw moment control strategies on stability enhancement in electric vehicles.

Despite the existence of research on stability control in electric vehicles, it is still an open research subject. This paper proposes a controller that is formulated using a solution of an off-line optimization problem, and takes feedback from the acceleration signals instead of the velocity signals. Since the control actions are optimized off-line, the controller is highly computationally efficient. The proposed controller is developed such that the actual vehicle's motion is adjusted according to driver commands. Thus, it is necessary to accurately interpret how the driver intends to manipulate the vehicle's motion, so as to enable the controller to generate appropriate control actions. For this purpose, a driver command interpreter module was developed. The efficiency of the controller for vehicle handling and the effects of hardware communications on overall system performance are shown through experiments carried out by driving the car based on standard maneuvers. The details of the work are given in the following sections.

\section{CONTROL FORMULATION}

The dynamics of a vehicle is consequence of forces and moment acting on the center of gravity (C. G.) of a vehicle. It is known that the C.G. forces are direct resultants of the tire forces, adjusted by a driver through steering and gas/brake pedal. However, it is possible that the vehicle motion differs from what the driver intended due to different reasons such as insufficient contact between road and tire or excessive lateral acceleration. Thus, the objective of the proposed controller is to keep the vehicle on the deriver's intended directional motion while providing the driver with a normal driving experience.

Defining the desired C.G. forces as those generated in stable conditions, the discrepancy between the desired C.G. forces and the actual ones can be corrected by controlling tire forces. Hence, the proposed control methodology uses longitudinal tire forces as the control variables.
The C.G. force errors and longitudinal tire force vectors are defined as:

$$
\begin{gathered}
E=\left[F_{x}^{*}-F_{x}, F_{y}^{*}-F_{y}, G_{z}^{*}-G_{z}\right]^{T}=\left[E_{x}, E_{y}, E_{z}\right]^{T} \\
f=\left[f_{x 1}, f_{x 2}, f_{x 3}, f_{x 4}\right]^{T}
\end{gathered}
$$

Definitions of parameters are provided at the end of this paper. With neglecting variation of lateral tire forces due to lack of active steering system, the error between the desired C.G. forces and actual ones can be adjusted as follows:

$$
\begin{aligned}
& F_{x}^{*}-F_{x}(f+\delta f) \cong \\
& F_{x}^{*}-\left[F_{x}(f)+\frac{d F_{x}(f)}{d f} \delta f\right]=E_{x}-\frac{d F_{x}(f)}{d f} \delta f \\
& F_{y}^{*}-F_{y}(f+\delta f) \cong \\
& F_{y}^{*}-\left[F_{y}(f)+\frac{d F_{y}(f)}{d f} \delta f\right]=E_{y}-\frac{d F_{y}(f)}{d f} \delta f \\
& G_{z}^{*}-G_{z}(f+\delta f) \cong \\
& G_{z}^{*}-\left[G_{z}(f)+\frac{d G_{z}(f)}{d f} \delta f\right]=E_{z}-\frac{d G_{z}(f)}{d f} \delta f
\end{aligned}
$$

where, $\delta f$ is the vector of longitudinal tire force adjustment calculated by the control unit.

The following objective function to be minimized includes the weighted combination of error between actual and desired vehicle C.G. forces and control action:

$$
P=\frac{1}{2}\left[\left(E-A_{F} \delta f\right)^{T} W_{E}\left(E-A_{F} \delta f\right)+\delta f^{T} W_{d f} \delta f\right]
$$

where, $W_{E}$ and $W_{d f}$ are the semi-positive definite diagonal weight matrices on the C.G. force error and control action vectors, respectively, and $A_{F}$ is associated with vehicle configuration where for a four wheel drive vehicle is:

$$
A_{F}=\left[\begin{array}{c}
\frac{d F_{x}(f)}{d f} \\
\frac{d F_{y}(f)}{d f} \\
\frac{d G_{z}(f)}{d f}
\end{array}\right]=\left[\begin{array}{llll}
\frac{\partial F_{x}}{\partial f_{x 1}} & \frac{\partial F_{x}}{\partial f_{x 2}} & \frac{\partial F_{x}}{\partial f_{x 3}} & \frac{\partial F_{x}}{\partial f_{x 4}} \\
\frac{\partial F_{y}}{\partial f_{x 1}} & \frac{\partial F_{y}}{\partial f_{x 2}} & \frac{\partial F_{y}}{\partial f_{x 3}} & \frac{\partial F_{y}}{\partial f_{x 4}} \\
\frac{\partial G_{z}}{\partial f_{x 1}} & \frac{\partial G_{z}}{\partial f_{x 2}} & \frac{\partial G_{z}}{\partial f_{x 3}} & \frac{\partial G_{z}}{\partial f_{x 4}}
\end{array}\right]
$$

Since $W_{E}$ and $W_{d f}$ are semi-positive definite, the quadratic objective function (6) is positive definite as well. Thus, the condition for minimization of $P$ as a linear set of equations in the matrix form is: 


$$
\frac{\partial P}{\partial \delta f}=0
$$

The minimizing of the objective function $P$ results in:

$$
\delta f=\left[W_{d f}+\left(A_{F}^{T} W_{E}\right) A_{F}\right]^{-1}\left(A_{F}^{T} W_{E}\right) E
$$

Thus, the torque required to be added to the nominal torque provided by the driver is:

$$
\delta Q=\delta f . R_{t}
$$

The proposed controller has a closed-from solution for an off-line optimization problem thereby making its real-time implementation more effective than model-based controllers available in the literature. In addition, the controller feedbacks are acceleration signals instead of velocities. In practice, the acceleration signals may be measured accurately by accelerometers or Inertial Measurement Units (IMU). Since the controller takes feedback from acceleration instead of velocities, its performance can be studied through the concept of state-derivative feedback systems. For brevity of the paper, more details about the control structure, the stability of the closed loop system, and controller gain optimization are referred to [11]. Figure 1 represents the overall block diagram of the closed-loop control system.

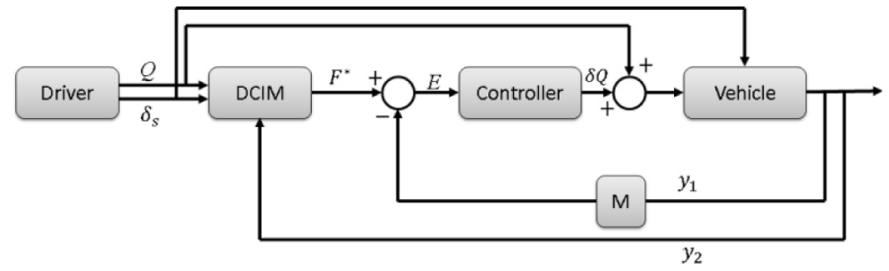

Figure 1. Block diagram of the overall close-loop system

In the above block diagram, $y_{1}=\left[\begin{array}{lll}\dot{v}_{x} & \dot{v}_{y} & \dot{r}\end{array}\right]^{T}$ is the vector of the output signals and $y_{2}=\left[\begin{array}{ll}v_{x} & \dot{v}_{y}\end{array}\right]^{T}$ are the measured signals as the input to DCIM which stands for the driver command interpreter module and its function is to map the driver's command signals to the target C.G. forces. The details of DCIM are explained in the next section.

\section{DRIVER COMMAND INTERPRETER}

In some references [12], [13] $],[14]$ this module has been defined as upper-controller which its function is to generate the desired signals so as to keep the vehicle in stable regions. However, this concept may result in over-restricting the driver in emergency cases. In such a case, the controller may keep the vehicle in stable region at cost of over-riding driver's control capability on the vehicle. Furthermore, in order to have the vehicle stable within the limits of both vehicle and road, the reference signals should be such that the vehicle can be reactive to commands of the driver. To address these issues, the objective of DCIM developed in this paper is defined to just generate target signals based on commands provided by driver in normal driving conditions.

A driver typically manipulates the vehicle through the steering wheel and gas/brake pedal in order to achieve intended directional motion. Since the objective of the controller is to provide the driver with the same driving experience as that on a dry road conditions with a normal tire characteristics, it is assumed that, in DCIM, the road surface coefficient, $\mu$, is equal to one and the tire characteristics are not subject to aging and wearing. Efficiency and accuracy are two conflict objectives which have to be considered in developing of DCIM. The module should be enough computationally efficient for real-time implementation while enough accurate to avoid over/under estimating driver's intent. With consideration of physical limitations, a nonlinear vehicle bicycle model is utilized to map the driver manipulation signals to controller reference signals (defined as desired C.G. forces) while maintaining reasonable limits to them. Then, when needed, the controller generates the proper control actions based on these data.

The following equations represent the dynamics of a vehicle bicycle model.

$$
\begin{aligned}
& F_{x}^{*}=f_{x f} \cos \left(\delta_{f}\right)-f_{y f} \sin \left(\delta_{f}\right)+f_{x r}+\left(r v_{y}+H_{R C} \phi \dot{r}\right) \\
& F_{y}^{*}=f_{x f} \sin \left(\delta_{f}\right)+f_{y f} \cos \left(\delta_{f}\right)+f_{y r}-\left(r v_{x}-H_{R C} \dot{\phi}\right) \\
& M_{z}^{*}=f_{x f} \sin \left(\delta_{f}\right) L_{f}+f_{y f} \cos \left(\delta_{f}\right) L_{f}-f_{y r} L_{r}+I_{x z} \ddot{\phi}
\end{aligned}
$$

The roll angle can be estimated by a single degree of freedom roll dynamic model:

$$
\ddot{\phi}=\frac{1}{I_{x}}\left[-m H_{R C}(\dot{v}+r u)+m g H_{R C} \phi-K_{\phi} \phi-C_{\phi} \dot{\phi}+I_{x z} \dot{r}\right]
$$

The dynamics of wheels are modeled as:

$$
\dot{\omega}_{i}=\frac{1}{I_{w}}\left(Q_{i}-f_{x i} R_{t}\right)
$$

where $i: f, r$. The tire forces $f_{x i}$, and $f_{y i}$ are calculated using a nonlinear combined slip tire model.

$$
\begin{aligned}
& f_{x i}=f_{X}\left(\kappa_{i}, F_{z i}\right) \cdot \phi_{x}\left(\kappa_{i}, \alpha_{i}\right) \\
& f_{y i}=f_{Y}\left(\alpha_{i}, F_{z i}\right) \cdot \phi_{y}\left(\kappa_{i}, \alpha_{i}\right)
\end{aligned}
$$

where

$$
f_{X}\left(\kappa_{i}, F_{z i}\right)=c_{1 x i} F_{z i} \sin \left[c_{2 x i} \tanh \left(c_{3 x i} \cdot \kappa_{i}\right)\right]
$$




$$
f_{Y}\left(\alpha_{i}, F_{z i}\right)=c_{1 y i} F_{z i} \sin \left[c_{2 y i} \tanh \left(c_{3 y i} . \kappa_{i}\right)\right]
$$

and

$$
\begin{aligned}
& \phi_{x}\left(\alpha_{i}, \kappa_{i}\right)=\left[1-\lambda_{x i} \operatorname{sech}\left(\gamma_{x i} \cdot \kappa_{i}\right) \tanh \left(\beta_{x i} \cdot \alpha_{i}\right)^{2}\right] \\
& \phi_{y}\left(\alpha_{i}, \kappa_{i}\right)=\left[1-\lambda_{y i} \operatorname{sech}\left(\gamma_{y i} \cdot \kappa_{i}\right) \tanh \left(\beta_{y i} \cdot \alpha_{i}\right)^{2}\right]
\end{aligned}
$$

and

$$
\begin{gathered}
\kappa_{i}=\frac{v-r \omega_{i}}{\max \left(v, r \omega_{i}\right)} \\
\alpha_{i}=\delta_{i}-\tan ^{-1}\left(\frac{v+L_{i} r}{u}\right)-C_{\text {roll }} \phi
\end{gathered}
$$

The weight of the vehicle is assumed to be equally distributed on the front and rear wheels.

$$
F_{z i}=\frac{m g}{2}
$$

Based upon the tire/road conditions, it is required to define an upper-bound on desired forces calculated in (11), (12), (13) in order to avoid generating excessive side and longitudinal slips. In steady state and stable conditions without side-slip, the upper-bound are defined as follows:

$$
\begin{gathered}
F_{x}^{*} \leq \mu \sum_{i=1}^{2}\left(\frac{Q_{i}}{R_{i}}\right) \\
F_{y}^{*} \leq \mu m g \\
M_{z}^{*} \leq I_{z} \frac{d}{d t}\left(\frac{a_{y}}{v_{x}}\right)
\end{gathered}
$$

For cases in which any of target signals goes greater than upper value, the upper bound will be considered as the target value.

\section{IMPLEMENTATION ISSUES AND SOLUTIONS}

Before compiling the controller codes onto the on-board vehicle microprocessor, some modifications and tunings are performed to solve some real-time implementation issues.

The first issue was associated with differences in the sample times of the DCIM and the sensors. The differences between these sample times make the closed-loop system prone to instability, due to errors in the DCIM outputs. It was noted that different sample times cause the inputs to the DCIM not to be updated properly. This issue was resolved by internally predicting the data for the missing sample-times.
As shown in figure 2, sensory data are fed to the DCIM at time $t$, while the calculated desired forces are produced at time $t+n . \Delta t$, where $\Delta t$ is the sampling time of the DCIM and $n$ is the number of missing sample times. Thus, the DCIM requires updating its parameters in this time span by running the built-in vehicle dynamics model. In this work, the sample time considered for the developed DCIM is smaller than the sample time for sensors and hardware communications.

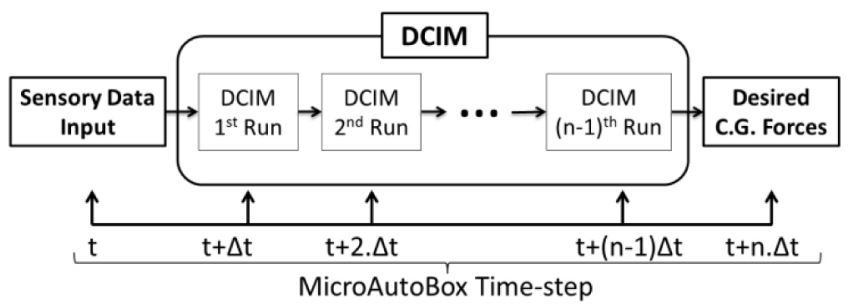

Figure 2. DCIM internal prediction

The second issue was related to existing noises in the outputs of the steering-column sensor and the wheels' optical sensors, which made the signals calculated by the DCIM noisy. In addition, low-frequency noises were observed in the vehicle's IMU signals, causing large discrepancies/errors in the C.G. forces of the proposed controller. These noises were removed by applying Chebyshev filters to the signals of both the DCIM and the IMU. The main reasons for choosing this filter for this application are its fast response time, and its more reliable real-time performance compared to other lowpass filters. As shown in figure 3 , this Chebyshev lowpass filter is free of ripple in the passband region, while there might be ripples in the stopband region. The gain of this filter is:

$$
G_{n}=\frac{\varepsilon}{\sqrt{1+\varepsilon^{2}}}
$$

where the ripple factor $(\varepsilon)$ is equal to:

$$
\varepsilon=\frac{1}{\sqrt{10^{0.1 \gamma_{\text {filter }-1}}}}
$$

The cutoff frequency $\left(F_{h}\right)$ of the filter is determined by the $-3 \mathrm{~dB}$ point of the filter magnitude response relative to the peak passband value, and the stopband edge frequency $\left(F_{c}\right)$ is related to $F_{h}$ by:

$$
F_{c}=F_{h} \cdot \cosh \left(\frac{1}{n} \cosh ^{-1} \frac{1}{\varepsilon}\right)
$$

This filter has steeper transition-region roll-off, but more nonlinear-phase response characteristics. 


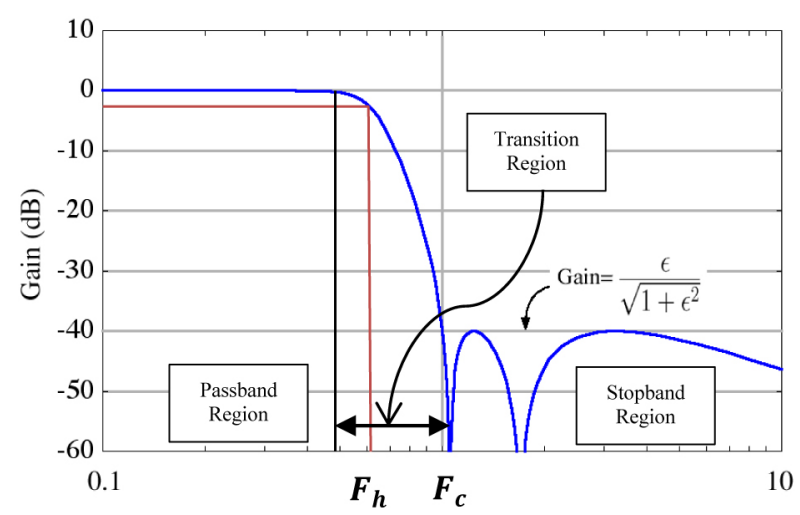

Figure 3. Chebyshev filter Bode diagram [15]

Although the system was affected by delay due to filter integration, the amount of delay is sufficiently small not to deteriorate the controller's stability and accuracy.

The last issue was related to using the angularacceleration signal as input to the controller. It was noted that the IMU provides yaw rate while the controlled required yaw acceleration. A $5^{\text {th }}$-order differentiator was used to solve this issue. To obtain the yaw acceleration, the last five data points plus the current yaw-rate values are needed. The formulation of the differentiator is:

$$
\dot{r}=\frac{16 U_{0}+U_{1}+10 U_{2}-10 U_{3}-6 U_{4}+9 U_{5}}{28 . \Delta d t}
$$

where

$$
U_{i}=r(t-i) \quad i=0,1, \ldots, 5
$$

\section{VEHICLE AND HARDWARE DESCRIPTION}

For road test experiments, a compact vehicle equipped with in-wheel electric motors was used. The controller code was compiled to a dSPACE MicroAutoBox installed on the vehicle. The hardware and software communicate with each other through the CAN bus and the dSPACE box. The inputs to the system are the wheels' steering angle and the gas/brake pedal position obtained from sensors in the car. The main drive system of the electric vehicle consists of an accelerator/ brake pedal, a traction electric motor, and batteries. In the following, a brief description of the hardware components integrated into the vehicle is given.

\section{Electric in-wheel motors}

Four Axial-flux, permanent magnet, synchronous AC Direct-Drive wheel motors are installed on the wheel hubs. Each motor has a maximum torque generation of $800 \mathrm{Nm}$ with $350 \mathrm{~A}$ and has $60 \mathrm{~kW}$ peak power at $1200 \mathrm{rpm}$. Figure 4 shows one of the motors installed on the vehicle. These motors are used to produce the traction torque requested by the driver and the adjustment torques requested by the integrated controller. It is noted that $\mathrm{AC}$ motors have the advantages of higher efficiency, higher power density, lower operating cost, lighter weight, and little to no need for maintenance, when compared with DC motors. Additionally, synchronous AC electric motors benefit from a smaller size, higher power density, lower cost, and higher efficiency in comparison with other available AC electric motors.

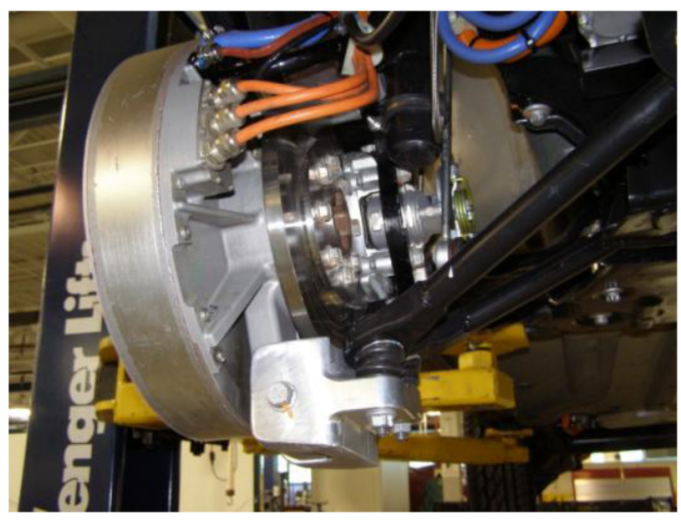

Figure 4. Electric in-wheel motor

\section{MicroAutoBox}

To run the vehicle code including the proposed controller, DCIM, filters, etc., a real-time rapid prototyping system with a $800 \mathrm{MHz}$ processor and $8 \mathrm{MB}$ main memory is used as the micro-processor. This stand-alone compact unit is ideal for installation on-board the vehicle. MicroAutoBox offers interfaces for all major automotive bus systems, such as CAN, LIN, and FlexRay. [1]

\section{CAN}

CAN stands for controller area network, and is a messagebased protocol that permits the electric hardware including the sensors and microcontrollers to communicate with each other without a host computer. The amount of wires required to connect the hardware in the vehicle can be reduced by using the CAN system. This robust system is high speed, low cost, and expandable. All communications between the electric vehicle MicroAutoBox and other hardware pass through the CAN. The main function of the CAN bus is to prioritize and to manage the received sensory signals and the outgoing control actions.

\section{Sensors}

The sensors used in the vehicle include a three-axial inertia measurement unit (IMU), dual potentiometers, and an encoder installed on the steering column to read the driver's steering hand wheel angle.

The dual potentiometers provide signals from the gas or brake pedals. These sensor are used to monitor the amount of total power being directed to the motors. The dual potentiometers are used as backup, in case the primary sensor malfunctions. This way the second sensor, which is mounted at a fixed distance from the first sensor, confirms the output 
value defined by the first sensor. In the case of any inconsistency, a safe predefined action will be sent to the system.

A digital frequency wheel speed output is provided by the connector of the wheel motor. It simulates a wheel speed signal that has sixty pulses per revolution. The frequency of the digital output is updated every 1 milliseconds. The digital output signal is pulled to $+12 \mathrm{~V}$ externally by a $3 \mathrm{~K}$ pull-up resistor and switched to ground by open collector transistors in the wheel motor driver, in order to generate the output signal.

\section{Batteries}

Two NiMHax 264-55ic batteries are installed on the vehicle for supplying energy to the front and rear motor wheels. Each battery has $1.8 \mathrm{Wh}$ power and results in an 18 $\mathrm{km}$ travel range. In addition, two $220 \mathrm{~V}, 30 \mathrm{~A}, 5 \mathrm{~kW}$ Manzanita Chargers are installed in order to re-charge the batteries. Figure 5 shows one of the batteries and chargers. In addition to these two batteries, an extra $12 \mathrm{~V}, 40 \mathrm{~A}$ power supply is used to power the auxiliary electrical devices such as the MicroAutoBox and sensors. This battery is also used as an emergency power unit in the case of an outage of the main two batteries.

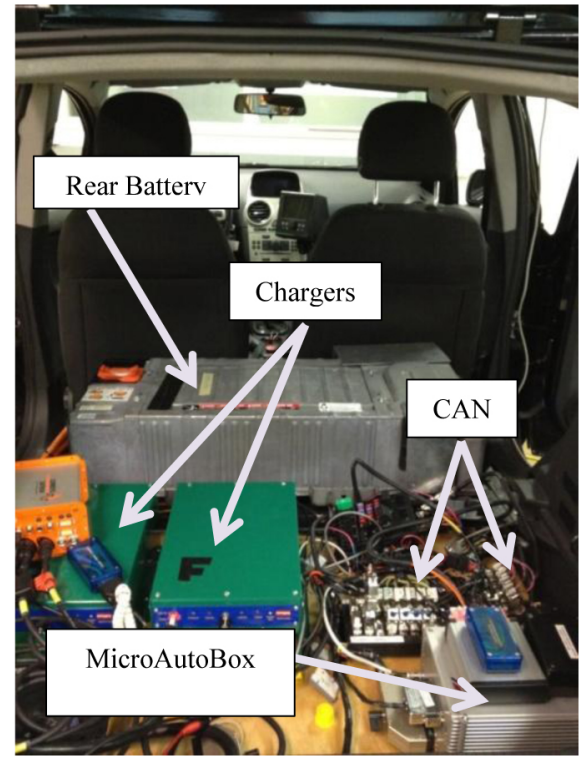

Figure 5. The hardware used for the electric vehicle

\section{DC-DC converter}

An on-board $300 \mathrm{~V}$ to $12 \mathrm{~V}$ DC-DC converter supply is placed in the vehicle to convert high-voltage power to lowvoltage power for the $12 \mathrm{~V}$ battery, and to keep this external power supply fully charged.

\section{$D C-A C$ inverter}

The main purpose of the inverter is to convert highvoltage $\mathrm{DC}$ power into $\mathrm{AC}$ propulsion power in order to drive the traction motors. It is also capable of acting as a rectifier to convert the AC power to high voltage DC power during regenerative braking mode. The minimum normal operating voltage of the PIM is 240 Volts.

\section{Friction brake}

Two standard brake calipers from Delta Brake Co. (part no. 10365717-8) are installed on the front wheels, which are connected through the brake fluid hydraulic line to the electric/hydraulic brake (EHB) system. This is an electric over hydraulic braking system using the electric power from the power supply. The negative torque generated from driver's brake pedal is maintained by the signal sent from Pulse-Width Module (PWM) to this EHB system connected to the friction pads on the wheels. This negative torque is also fed to the DCIM as an input.

The reason to have the friction brakes mounted in the front wheels is to improve the steerability of the vehicle during braking.

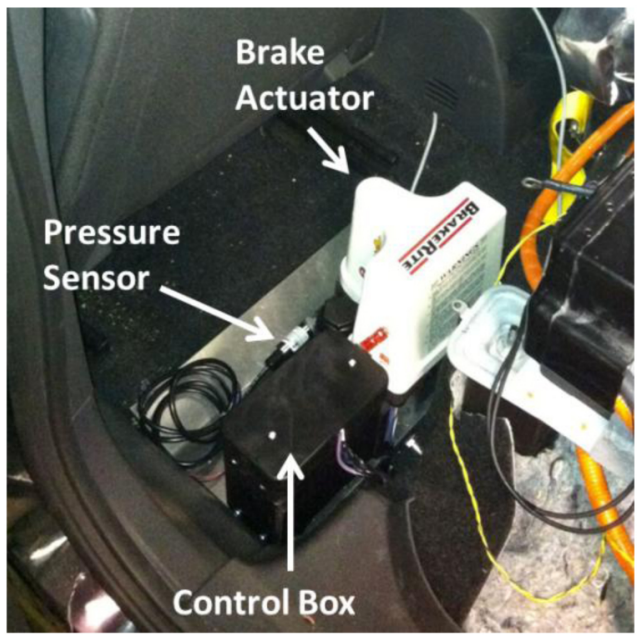

Figure 6. Friction brake actuator and control box

\section{Diagnostic interface panel}

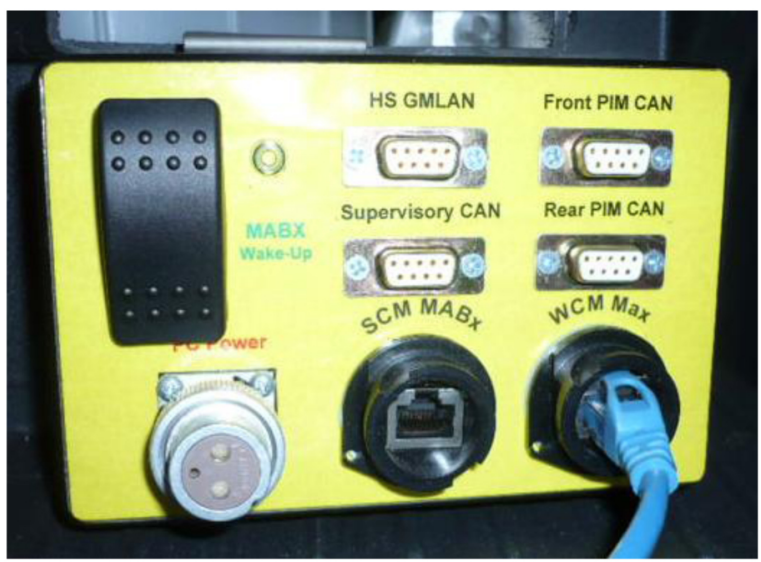

Figure 7. Diagnostic Interface Panel 
This unit is located in passenger glove compartment for easy access to MicroAutoBox for code compilation and other setup. This way, long wires for connecting the laptop from passenger seats to the trunk (MicroAutobox location) will be eliminated. Figure 7 shows this communicating unit.

\section{DCIM VERIFICATION}

In this section, the accuracy of DCIM is verified by CarSim simulations.

As mentioned already, the function of DCIM is to generate the dynamic response of vehicle in normal driving conditions such as dry road and perfect tire characteristics. According to the physical parameters and properties of Opel Corsa, a proper model of the vehicle is developed in CarSim. The road surface coefficient assumed to be as dry road conditions, $\mu=1$.

CarSim inputs are steering wheel angle and driving torques as shown in Figures 8 and $\underline{9}$, while the C.G. forces obtained from CarSim and DCIM are compared through figure 10 , figure 11 , figure 12 .

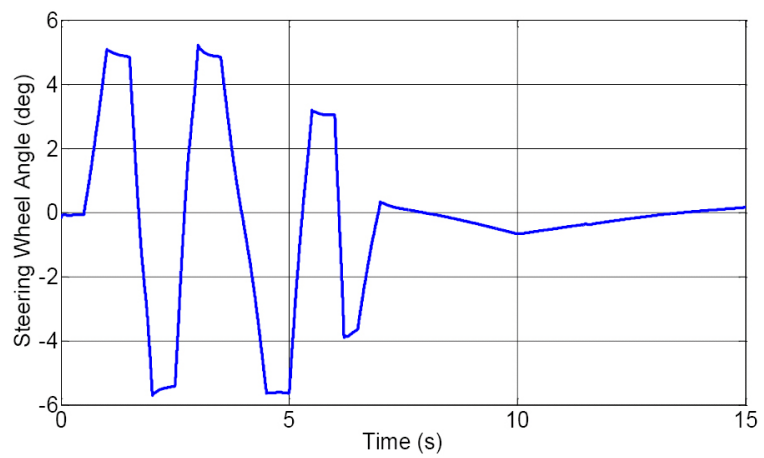

Figure 8. Driver's steering input

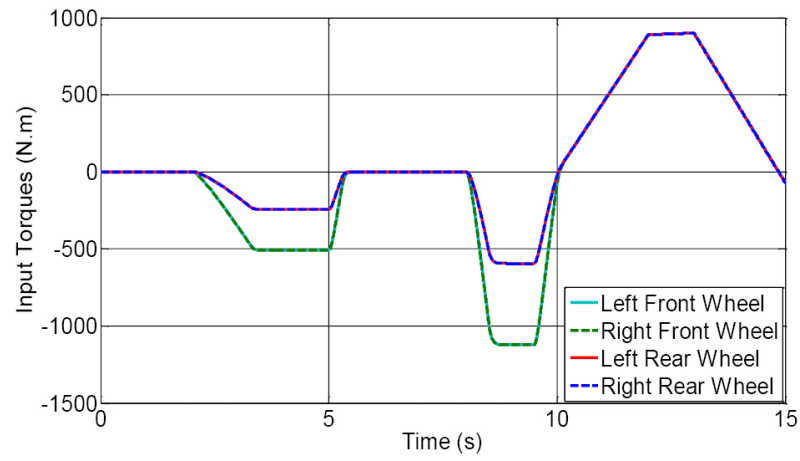

Figure 9. Driver's torque input

Initial velocity of the vehicle model is $100 \mathrm{~km} / \mathrm{hr}$. The target path is assumed to be the path that the driver would like to follow on a dry road. As shown in these figures, DCIM estimate the C.G. forces with reasonable accuracy, based on the driver's inputs. For brevity of the paper, more simulation results about the DCIM and closed-loop system performance are referred to Ref. [11]

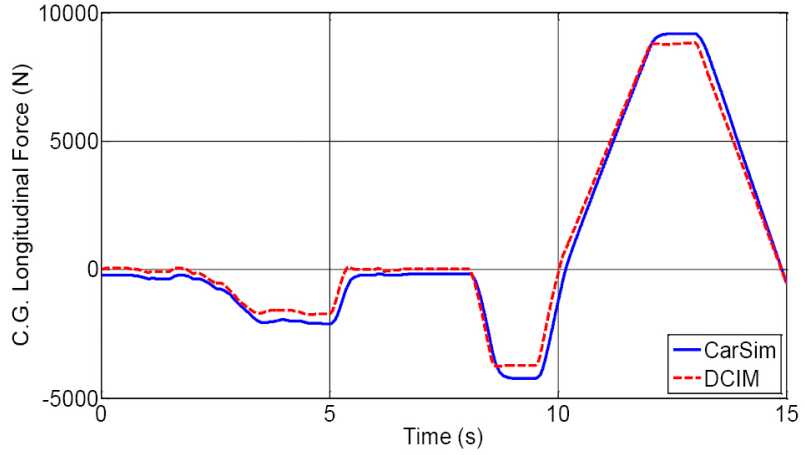

Figure 10. Comparison of longitudinal forces

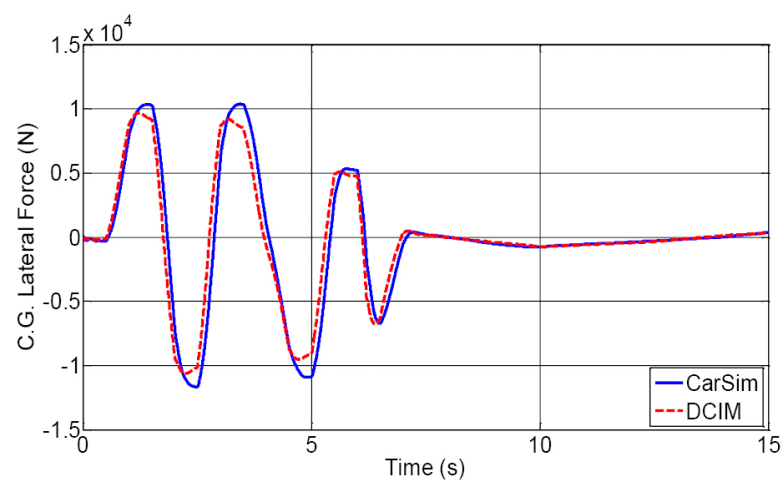

Figure 11. Comparison of laterl forces

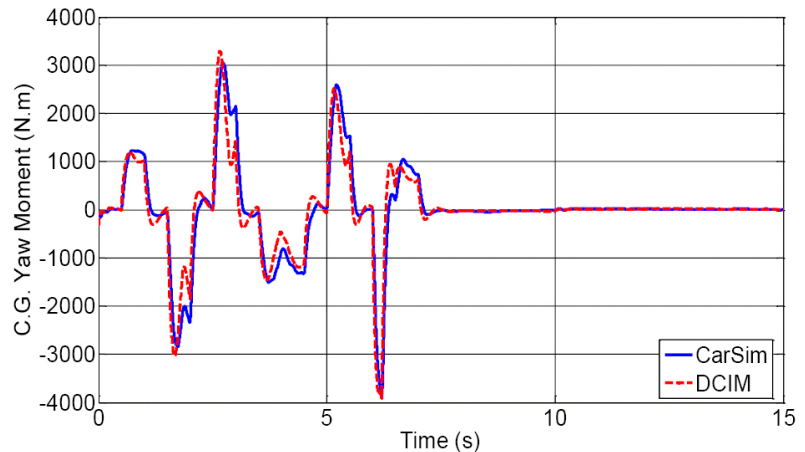

Figure 12. Comparison of yaw moments

\section{EXPERIMENTAL RESULTS}

In this section, the accuracy of the DCIM, real-time controller performance, and hardware communications are investigated through experiments.

\section{DCIM real-time performance}

For investigation of the DCIM, it is assumed that the controller is off and the DCIM signals are compared with those obtained from IMU. Figures 13 and 14 show the driver inputs during the test, while figure 15 , figure 16 , figure 17 show the results received from vehicle IMU and DCIM 
during real time standard double-lane-change scenario at 60 $\mathrm{km} / \mathrm{h}$ speed and subject to dry perfect road conditions.

These figures clearly show the accuracy of the DCIM in generating the desired forces for the real vehicle. Offset in longitudinal forces are because of some difference between the assumed drag forces and the actual one. The drag force assumed in DCIM is linear proportional to the velocity, while the actual drag force is more complicated. The other source of discrepancy is using the simplified rolling resistance force in DCIM.

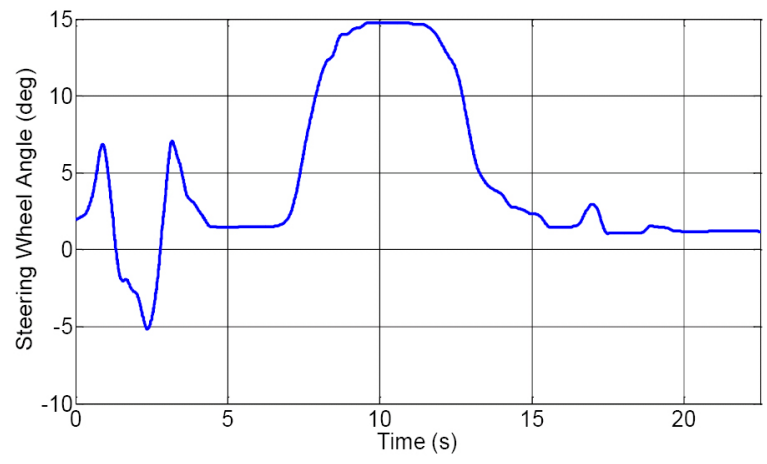

Figure 13. Driver's steering input

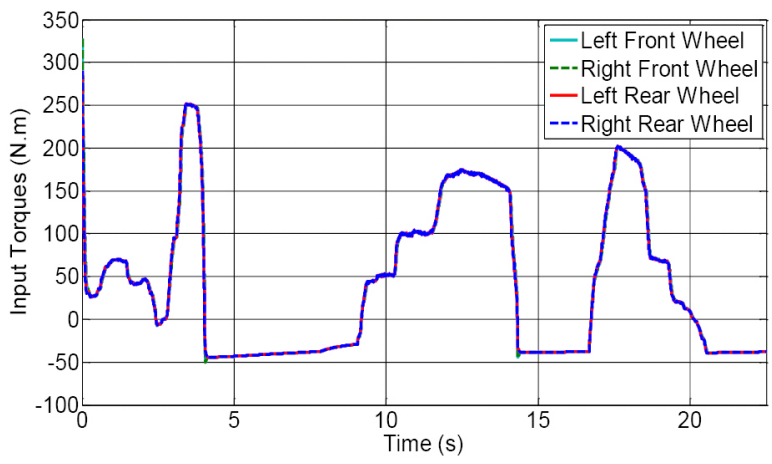

Figure 14. Driver's torque input

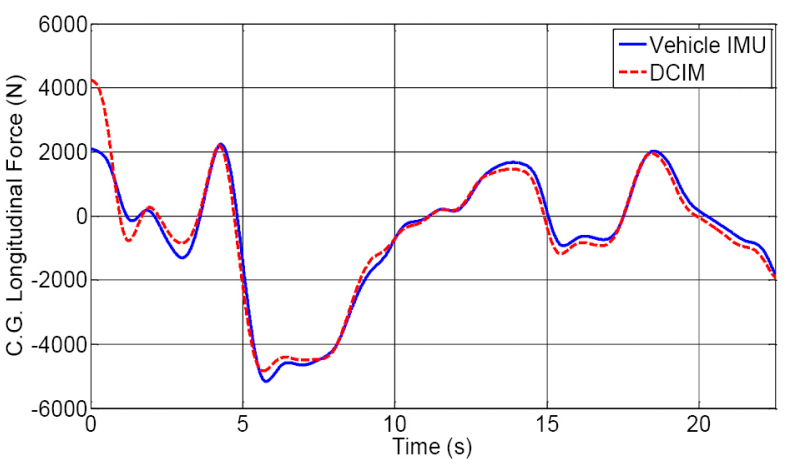

Figure 15. Comparison of longitudinal forces

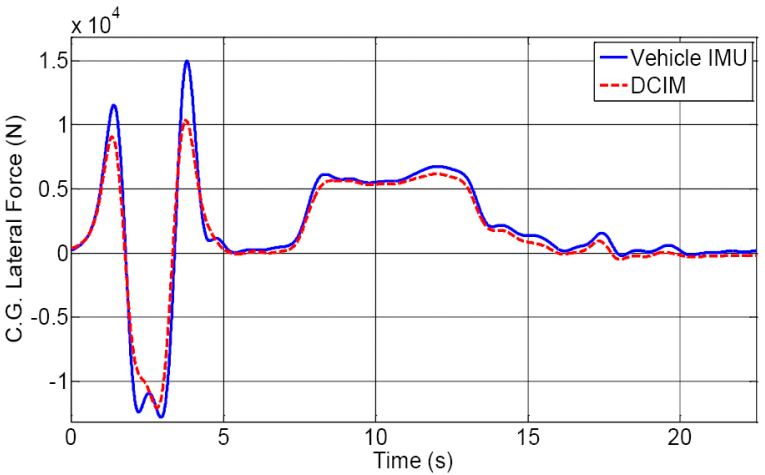

Figure 16. Comparison of lateral forces

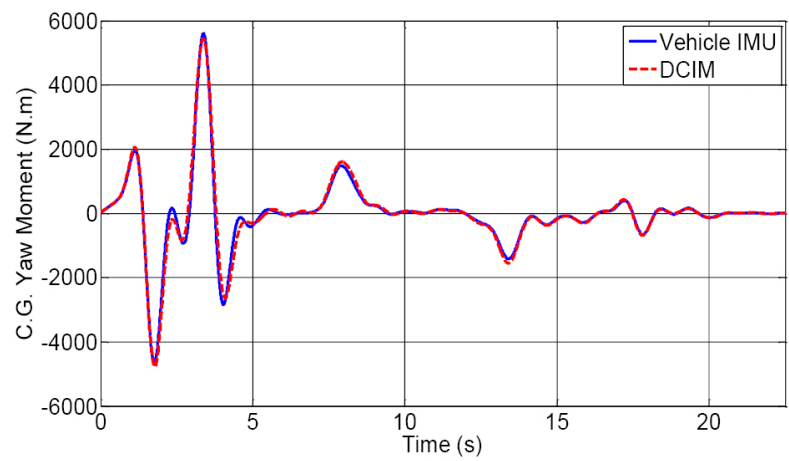

Figure 17. Comparison of yaw moments

\section{Controller real-time performance}

This sub-section shows the controller's overall performance through results obtained from two different double-lane-change tests, with and without controller. Figure 18, Figure 19, Figure 20, Figure 21, Figure 22, Figure 23, Figure 24, Figure 25, Figure 26, Figure 27, Figure 28, figure 29 illustrate the results where the road coefficient of friction was less than 1 , since it was raining conditions just before the experiments.

Figure 18 depicts the driver's effort on the steering hand wheel in the cases of on and off controller. As shown, the driver needs to put more effort on the steering to keep the vehicle on the target path, when the controller is not activated.

Figure 19, Figure 20, figure 21 compares the vehicle C.G. longitudinal force, lateral force, and yaw moment, respectively, while figure 22 , figure 23 , figure 24 show the longitudinal, lateral, and yaw moment errors. As illustrated, the controller improves vehicle handling performance and stability by reducing the peak values and settling down the dynamic response and errors to zero faster.

Figures 25 and 26 show the slip and heading angles, respectively. As shown, when the controller is activated, these angles also become smaller, which represent the more stability and better handling performance of the vehicle on the road compared to passive system. 
Figure 28 and figure 29 show the total torques applied at front left and rear right wheels, with and without controller. When the controller is on, the total torque is the summation of torques applied by the driver and the torque generated by the controller.

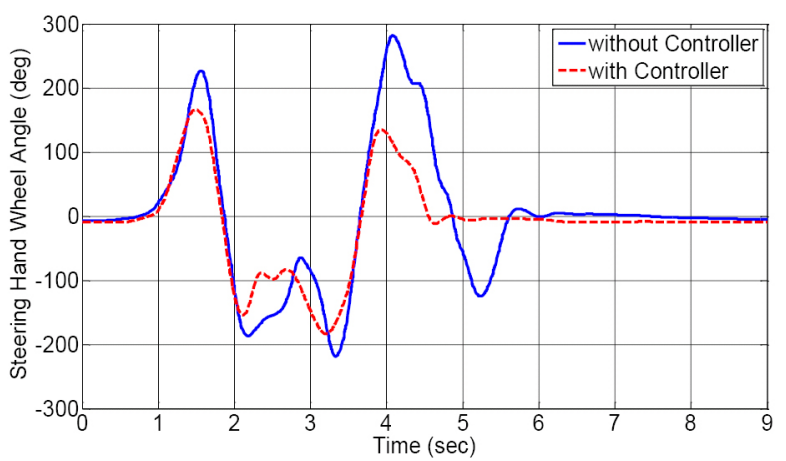

Figure 18. Comparison of driver's steering inputs

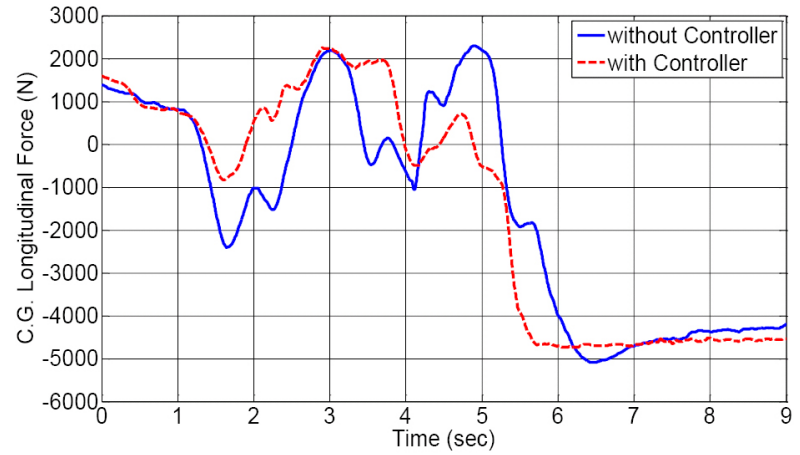

Figure 19. Comparison of longitudinal forces

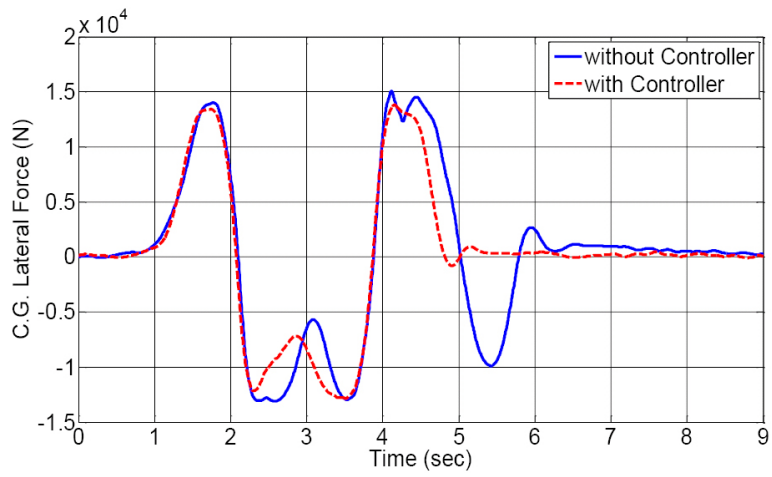

Figure 20. Comparison of lateral forces

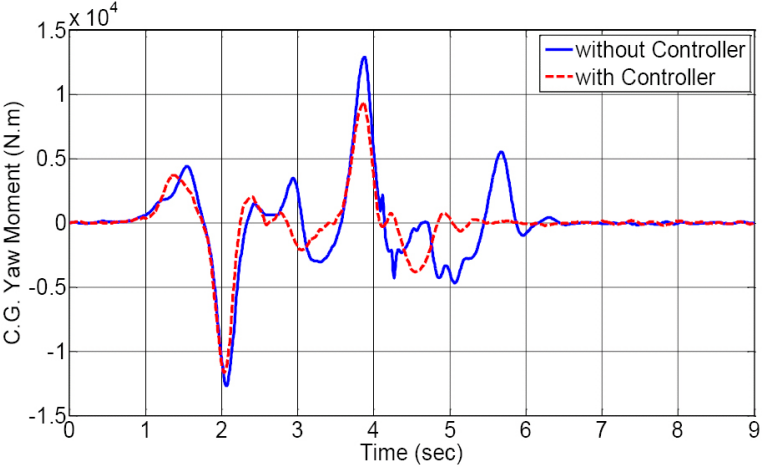

Figure 21. Comparison of yaw moments

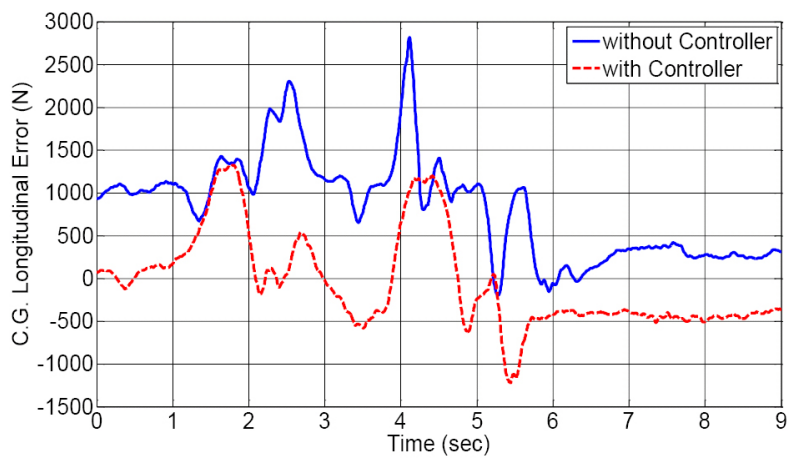

Figure 22. Comparison of errors in longitudinal forces

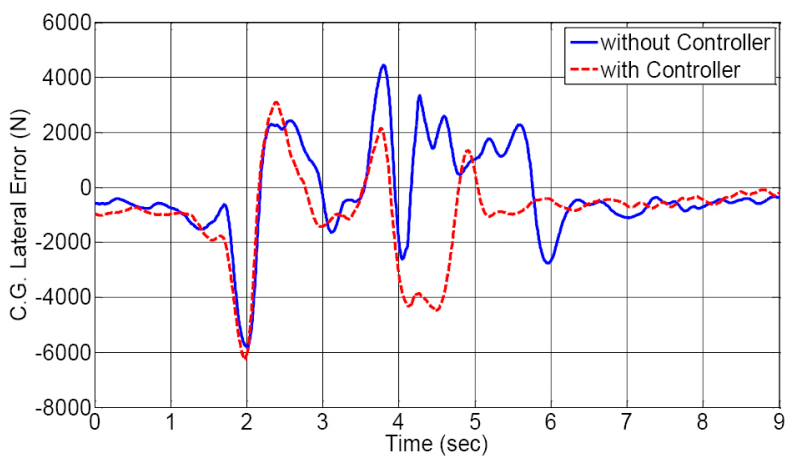

Figure 23. Comparison of errors in lateral forces

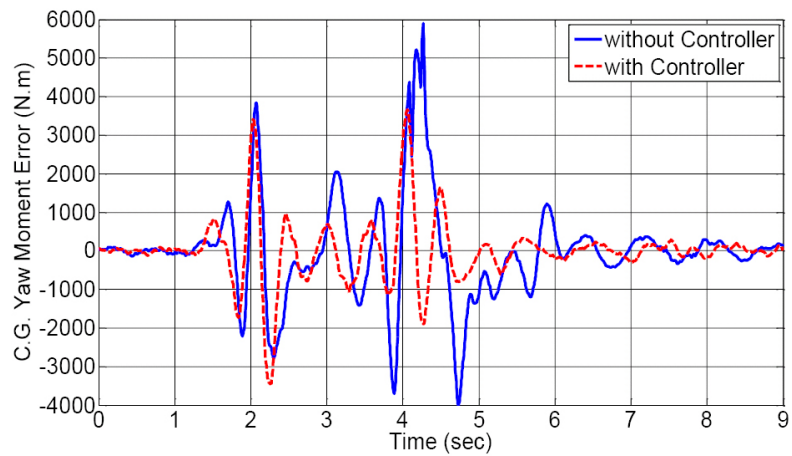

Figure 24. Comparison of errors in yaw moments 


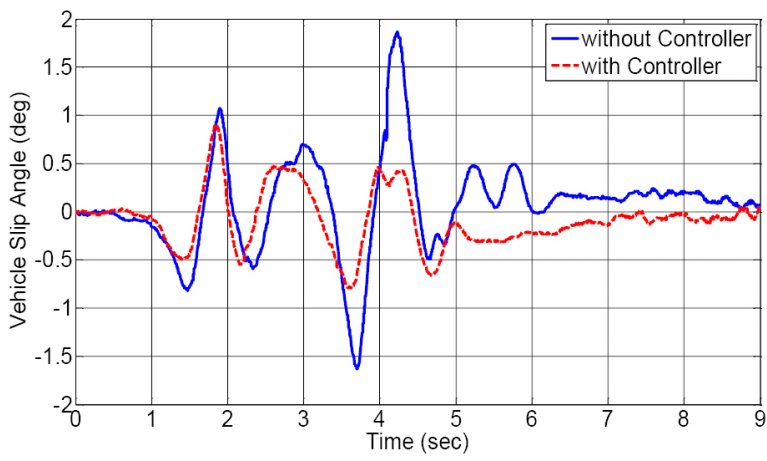

Figure 25. Comparison of vehicle slip angles

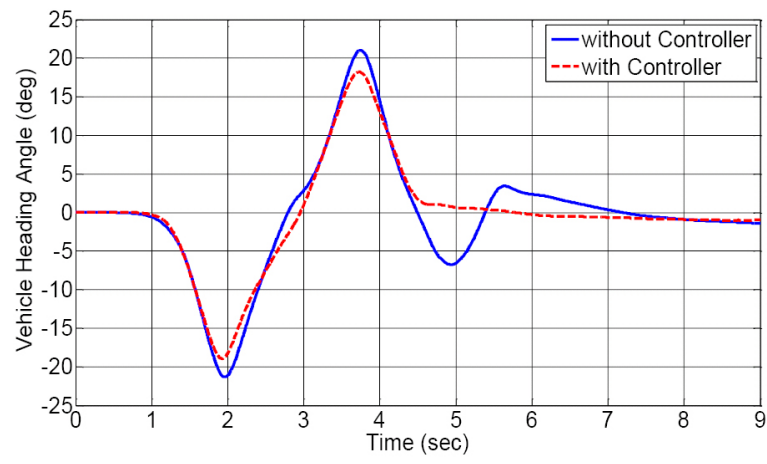

Figure 26. Comparison of vehicle heading angles

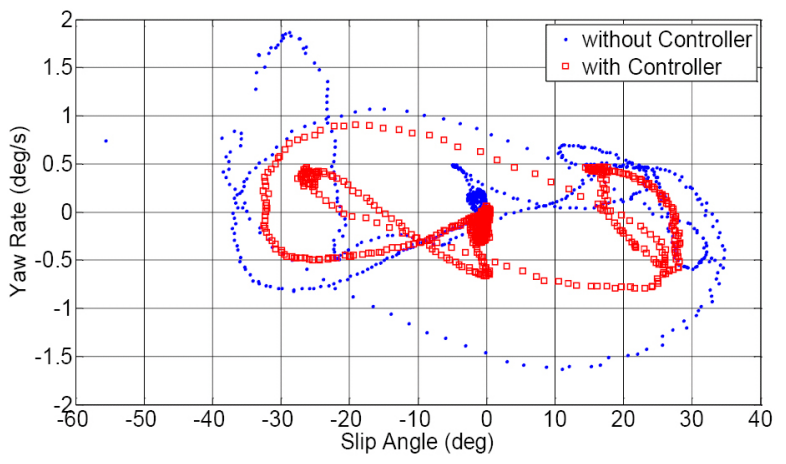

Figure 27. Comparison of yaw-rate vs. slip angle

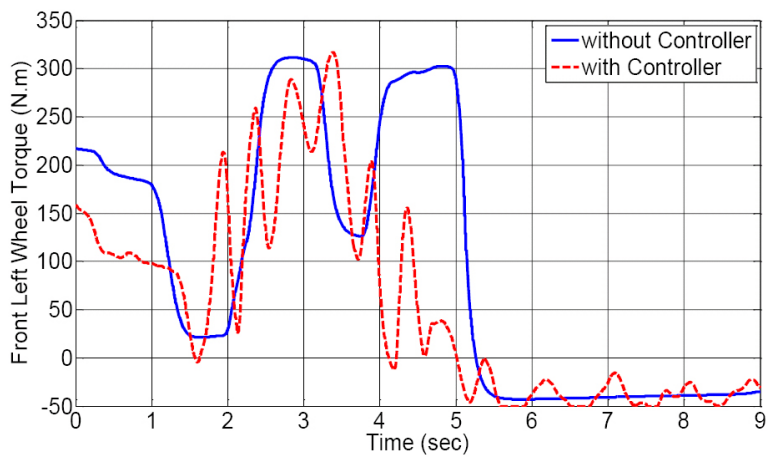

Figure 28. Comparison of front left wheel torques

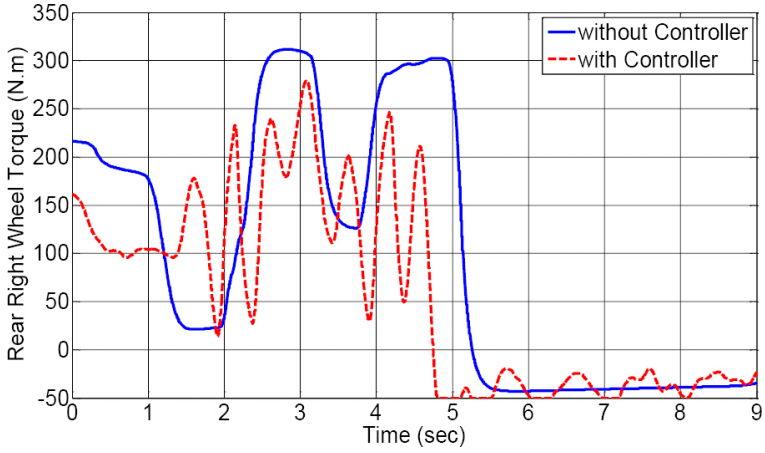

Figure 29. Comparison of rear right wheel torques

\section{CONCLUSIONS}

The paper proposed a novel torque vectoring control strategy to assist a driver in handling a vehicle in unexpected conditions. With a closed-form structure, the controller was formulated by solving an off-line optimization problem. In order to interpret the driver's intention and to generate proper control actions, a module was developed using an accurate bicycle vehicle model. The accuracy of each of the driver command interpreter module and the controller was verified through experiments. An in-wheel electric vehicle equipped with various sensors and hardware was used for the experiments. The results show the stability of the overall system and also a significant enhancement of the vehicle's performance by the controller.

\section{REFERENCES}

1. Chan, C. C., "An Overview of Electric Vehicle Technology," Proceedings of the IEEE, Vol. 81(9): 1202-1212, 1993, doi: 10.1109/5.237530.

2. Hori, Y., "Future Vehicle Driven by Electricity and Control-Research on Four-Wheel-Motored "UOT Electric March II"," IEEE Transactions on Industrial Electronics, Vol. 51(5): 954-962, 2004, doi: 10.1109/TIE. 2004.834944

3. Jain, M., Williamson, S. S., "Suitability Analysis of In-Wheel Motor Direct Drives for Electric and Hybrid Electric Vehicles," IEEE Electrical Power \& Energy Conference, 2009, doi: 10.1109/EPEC. 2009.5420886 .

4. Zhao, Y., Zhang, Y., Zhao, Y., "Stability Control System for Four-InWheel-Motor Drive Electric Vehicle," $6^{\text {th }}$ International Conference on Fuzzy Systems and Knowledge Discovery, Vol. 4: 171-175, 2009, doi: 10.1109/FSKD.2009.582.

5. Mutoh, N., Kazama, T., Takita, K., "Driving Characteristics of an Electric Vehicle System With Independently Driven Front and Rear Wheels," IEEE Transactions on Industrial Electronics, Vol. 53(3): 803-813, 2006, doi: 10.1109/TIE.2006.874271.

6. Mutoh, N., Hayano, Y., Yahagi, H., Takita, K., "Electric Braking Control Methods for Electric Vehicles With Independently Driven Front and Rear Wheels," IEEE Transactions on Industrial Electronics, Vol. 54(2): 1168-1176, 2007, doi: 10.1109/TIE.2007.892731.

7. Hori, Y., Toyoda, Y., Tsuruoka, Y., "Traction Control of Electric Vehicle: Basic Experimental Results Using the Test EV "UOT Electric March"," IEEE Transactions on Industry Applications, Vol. 34(5): 1131-1138, 1998, doi: 10.1109/28.720454.

8. Huang, Q., Huang, Z., Zhou, H. "Nonlinear Optimal and Robust Speed Control for a Light-Weighted All-Electric Vehicle," IET Control Theory Applications, Vol. 3(4): 437-444, 2009, doi: 10.1049/iet-cta.2007.0367.

9. Kim, D., Hwang, S., Kim, H., "Vehicle Stability Enhancement of FourWheel-Drive Hybrid Electric Vehicle Using Rear Motor Control," IEEE Transactions on Vehicular Technology, Vol. 57(2): 727-735, 2008, doi: 10.1109/TVT.2007.907016.

10. Sakai, S., Sado, H., Hori, Y., "Motion Control in an Electric Vehicle with Four Independently Driven In-Wheel Motors," IEEE/ASME 
Transactions on Mechatronics, Vol. 4(1): 9-16, 1999, doi: $10.1109 / 3516.752079$.

11. Fallah, M. S., Khajepour, A., Fidan, B., Litkouhi, B., and Chen, S. H., "Vehicle Optimal Torque Vectoring using State-Derivative Feedback and Linear Matrix Inequality", IEEE Transactions on Vehicular Technology, Accepted for publication, 2012.

12. Tseng, H. E., Ashrafi, B., Madau, D., and Brown, T. A., "The Development of Vehicle Stability Control at Ford", IEEE/ASME Trans. on Mechatronics, vol. 4(3): 223-234, 1999, doi: 10.1109/3516.789681.

13. Geng, C., Mostefai, L., Denai, M. Hori, Y "Direct Yaw-Moment Control of an In-Wheel-Motored Electric Vehicle Based on Body Slip Angle Fuzzy Observer," IEEE Transactions on Industrial Electronics, Vol. 56(5): 1411-1419, 2009, doi: 10.1109/TIE.2009.2013737.

14. Fujimoto, H., Tsumasaka, A., Noguchi, T., "Direct Yaw-Moment Control of Electric Vehicle Based on Cornering Stiffness Estimation," $31^{\text {st }}$ Annual Conference of IEEE Industrial Electronics Society, 2005, doi: $10.1109 /$ IECON.2005.1569321.

15. Absolute Astronomy, "Chebyshev Filter," http:// www.absoluteastronomy.com/topics/Chebyshev filter

16. dSPACE International, "MicroAutoBox Hardware," http:// www.dspace.de/shared/data/pdf/flyer2008/

dspace 2008 microautobox en pi480.pdf, Sep. 2008.

\section{CONTACT INFORMATION}

Abtin Athari, Corresponding Author

E3x-4102, Department of Mechanical and Mechatronics Engineering

University of Waterloo, 200 University Ave.

West, Waterloo, ON N2L 3G1, Canada.

a2athari@uwaterloo.ca

\section{ACKNOWLEDGMENTS}

The authors would like to acknowledge the financial support of Automotive Partnership Canada, Ontario Research Fund, and also technical and financial support of General Motors in this work.

\section{DEFINITIONS}

$\boldsymbol{\alpha}_{\boldsymbol{i}}$ - Tire slip angle $(\mathrm{rad})$

$\boldsymbol{\beta}_{\boldsymbol{x} i}$ - Constant parameter of tire model

$\boldsymbol{\beta}_{\boldsymbol{y} i}$ - Constant parameter of tire model

$\boldsymbol{\kappa}_{\boldsymbol{i}}$ - Tire slip ratio

$\phi$ - Roll angle ( $\mathrm{rad})$

$\dot{\phi}$ - Roll rate $(\mathrm{rad} / \mathrm{s})$

$\ddot{\phi}$ - Roll acceleration $\left(\mathrm{rad} / \mathrm{s}^{2}\right)$

$\lambda_{x i}$ - Constant parameter of tire model

$\lambda_{y i}$ - Constant parameter of tire model

$\gamma_{x i}$ - Constant parameter of tire model

$\gamma_{y i}$ - Constant parameter of tire model

$\boldsymbol{\delta}_{\boldsymbol{i}}$ - Road wheel angle ( $\mathrm{rad}$ )

$c_{1 x i}$ - Constant parameter of tire model

$\boldsymbol{c}_{2 x i}$ - Constant parameter of tire model

$c_{3 x i}$ - Constant parameter of tire model

$c_{1 y i}$ - Constant parameter of tire model $\boldsymbol{c}_{2 y \boldsymbol{i}}$ - Constant parameter of tire model

$c_{3 y i}$ - Constant parameter of tire model

$\boldsymbol{f}_{\boldsymbol{x} \boldsymbol{i}}$ - Longitudinal tire force $(N)$

$\boldsymbol{f}_{\boldsymbol{y} \boldsymbol{i}}$ - Lateral tire force $(N)$

$\boldsymbol{g}$ - Gravity acceleration $\left(\mathrm{m} / \mathrm{s}^{2}\right)$

$\boldsymbol{m}$ - Mass of the vehicle $(\mathrm{Kg})$

$\boldsymbol{r}$ - Vehicle yaw rate $(\mathrm{rad} / \mathrm{s})$

$\dot{r}$ - Vehicle yaw acceleration $\left(\mathrm{rad} / \mathrm{s}^{2}\right)$

$\boldsymbol{v}_{\boldsymbol{x}}$ - Longitudinal vehicle velocity $(\mathrm{m} / \mathrm{s})$

$\dot{v}_{x}$ - Longitudinal vehicle acceleration $\left(\mathrm{m} / \mathrm{s}^{2}\right)$

$\boldsymbol{v}_{\boldsymbol{y}}$ - Lateral vehicle velocity $(\mathrm{m} / \mathrm{s})$

$\dot{v}_{y}$ - Lateral vehicle acceleration $\left(\mathrm{m} / \mathrm{s}^{2}\right)$

$\boldsymbol{C}_{\text {roll }}$ - Roll Steering coefficient ( $\left.\mathrm{rad} / \mathrm{rad}\right)$

$\boldsymbol{C}_{\boldsymbol{\phi}}$ - Roll damping coefficient ( $\left.N . \mathrm{s} / \mathrm{rad}\right)$

$\boldsymbol{F}_{\boldsymbol{x}}$ - Actual longitudinal vehicle C.G. force $(N)$

$F_{x}^{*}$ - Desired longitudinal vehicle C.G. force $(N)$

$\boldsymbol{F}_{\boldsymbol{y}}$ - Actual lateral vehicle C.G. force $(N)$

$F_{y}^{*}$ - Desired lateral vehicle C.G. force $(N)$

$\boldsymbol{H}_{\boldsymbol{R} \boldsymbol{C}}$ - Height of roll center $(\mathrm{m})$

$\boldsymbol{I}_{\boldsymbol{z}}$ - Yaw moment of inertia about the $z$ axis $\left(\mathrm{Kg} \cdot \mathrm{m}^{2}\right)$

$\boldsymbol{K}_{\boldsymbol{\phi}}$ - Roll stiffness ( $\left./ \mathrm{rad}\right)$

$\boldsymbol{L}_{\boldsymbol{i}}$ - Distance from the vehicle C.G. to its axle $(\mathrm{m})$

$\boldsymbol{M}_{\boldsymbol{z}}$ - Actual yaw moment acting on vehicle C.G. (N.m)

$M_{z}^{*}$ - Desired yaw moment acting on vehicle C.G. (N.m)

$\boldsymbol{Q}$ - Driver torque vector input (N.m)

$\boldsymbol{Q}_{\boldsymbol{i}}$ - Torque acting on wheel (N.m)

$\boldsymbol{n}$ - Filter order

$\boldsymbol{F}_{\boldsymbol{h}}$ - Filter cutoff frequency $(\mathrm{Hz})$

$\boldsymbol{F}_{\boldsymbol{c}}$ - Filter stopband edge frequency $(\mathrm{Hz})$

$\gamma_{\text {filter }}$ - Filter stopband ripple magnitude $(d B)$

$\varepsilon$ - Filter ripple factor

$\boldsymbol{G}_{\boldsymbol{n}}$ - Filter gain (dB) 